\title{
Microcrustáceos planctónicos y características limnológicas de dos lagunas pampeanas (Buenos Aires, Argentina)
}

\author{
Emilio Javier Garibotti ${ }^{1, *}$, Patricia Marta Cervellini ${ }^{2}$, María Cintia Piccolo ${ }^{1,3}$ \\ ${ }^{1}$ Instituto Argentino de Oceanografía-CONICET, C.C. 804, B8000FWB, Bahía Blanca, Provincia de Buenos \\ Aires, Argentina. \\ ${ }^{2}$ Depto. Biología, Bioquímica y Farmacia, Universidad Nacional del Sur. San Juan 670, B8000DIC, Bahía Blan- \\ ca, Provincia de Buenos Aires, Argentina. \\ ${ }^{3}$ Depto. Geografía y Turismo, Universidad Nacional del Sur. 12 de octubre y San Juan, C.P. (8000) Bahía Blanca, \\ Provincia de Buenos Aires, Argentina.
}

* Autor responsable de la correspondencia: garibotti@ criba.edu.ar

Recibido: 11/10/08

Aceptado: 7/1/08

\begin{abstract}
Planktonic microcrustaceans and limnological characteristics of two pampean lagoons (Buenos Aires, Argentina)

In the Pampean Region, there are numerous lagoons of diverse forms, sizes and hydrography. The knowledge about these ecosystems in these lagoons is limited, especially about the plankton present in them. For this reason, the planktonic microcrustaceans and their relationship with the limnological conditions have been studied for two lagoons of the southwest of the Buenos Aires province, Argentina (Unamuno and Calderón lagoons) between October and December of 2006. A study site was selected for each lagoon. Every fifty days biological and physico-chemical samples were taken and environmental parameters were measured. Seven taxa were registered. The density fluctuated between 221 and $1703 \mathrm{ind} / \mathrm{l}$ in Unamuno and between 41 and 1401 ind/l in Calderón. Moina eugeniae Olivier 1954 and Boeckella poopoensis Marsh 1906 were the most abundant species, the absence of rotifers was obvious in both lagoons. Few species dominated and the individuals showed great size. The salinity was $0.39 \mathrm{~g} / \mathrm{l}$ in Unamuno and $1.69 \mathrm{~g} / \mathrm{l}$ in Calderón so they were characterized as oligohalines. High values of suspended sediments were registered in Calderón. Both lagoons presented low concentrations of silicate and nitrate, and high concentrations of nitrites and phosphates. The silicate concentrations were different between both lagoons. The dominant ions were $\mathrm{Na}^{+}$and $\mathrm{Cl}^{-}$, so they can be classified as bicarbonate sodium chlorinates. The concentrations of chlorophyll $a$ were important indicating a high algal biomass. These lagoons were different regarding their depth and the concentration of nutrients and ions, as well as the richness and abundance of species. It was clear that the abiotic factors, such as as salinity, were influential over the specific composition. This study offers information on the limnologic, physical, and chemical conditions of two lagoons of the Pampean Region and on the planktonic microcrustaceans that live in these ecosystems.
\end{abstract}

Key words: Zooplankton, lagoons, physical-chemical parameters, Argentina.

\section{RESUMEN}

Microcrustáceos planctónicos y características limnologicas de dos lagunas pampeanas (Buenos Aires, Argentina)

En la Región Pampeana se localizan numerosas lagunas de diversas formas, tamaños e hidrografía. Es limitado el conocimiento que se tiene sobre estos ecosistemas y en particular del plancton presente en ellas. Por este motivo, se han estudiado los microcrustáceos planctónicos y su relación con las condiciones limnológicas para dos lagunas del sudoeste de la provincia de Buenos Aires, Argentina (Laguna Unamuno y Laguna Calderón) entre octubre y diciembre de 2006. Se seleccionó un sitio de muestreo para cada laguna. Cada 15 días se obtuvieron muestras biológicas y fisicoquímicas y se midieron parámetros ambientales. Fueron registrados siete taxones. La densidad fluctuó entre 221 y 1703 ind/l en la Laguna Unamuno y entre 41 y 1401 ind/l en la Laguna Calderón. Las especies más abundantes en las dos lagunas fueron Moina eugeniae Olivier 1954 y Boeckella poopoensis Marsh 1906, la ausencia de rotíferos se manifestó para ambos cuerpos de agua. Dominaron pocas especies y los individuos presentaron gran tamaño. La salinidad fue $0.39 \mathrm{~g} / \mathrm{l}$ en la Laguna Unamuno y $1.69 \mathrm{~g} / \mathrm{l}$ en la Laguna Calderón, por lo que se las pudo caracterizar como oligohalinas. En la Laguna Calderón se registraron elevados valores de sedimento en suspensión. Las lagunas presentaron bajas concentraciones de nitratos y de silicatos y altas concentraciones 
de nitritos y de fosfatos. Las concentraciones de silicatos fueron diferentes para ambas lagunas. Los iones dominantes fueron $\mathrm{Na}^{+}$y $\mathrm{Cl}^{-}$, comportándose como bicarbonatadas sódicas cloruradas. Las concentraciones de clorofila a fueron importantes indicando una alta biomasa algal. Estas lagunas se diferenciaron en cuanto a su profundidad y a la concentración de nutrientes e iones, así como en la riqueza y abundancia de especies. Se vio que los factores abióticos, como el caso de la salinidad, influenciaron sobre la composición específica. Este estudio brinda información sobre las condiciones limnológicas, físicas y químicas de dos lagunas de la Región Pampeana y sobre los microcrustáceos planctónicos que habitan en estos ecosistemas.

Palabras clave: Zooplancton, lagunas, parámetros fisicoquímicos, Argentina.

\section{INTRODUCCIÓN}

Las lagunas del extenso territorio argentino se pueden agrupar dentro de regiones geográficas muy diferentes entre sí. Las distintas características fisicoquímicas y la amplia variación de sólidos totales e iones disueltos en sus aguas denotan su extensa variabilidad. Los cuerpos de agua del correspondiente estudio se encuentran dentro de la llamada "Región de la Llanura ChacoPampeana" que cubre la porción central, norte y este de la Argentina (Drago y Quirós, 1996). Según Iriondo (2004) a esta zona se la denomina humedal pampeano y cubre una superficie de unos $100000 \mathrm{~km}^{2}$ de planicies de pampas, constituyendo una de las zonas de humedales más extensa de América del Sur. Muchas lagunas de esta zona son permanentes, generadas por procesos de deflación eólica, un número importante ha sido remodelado por acción fluvial y en algunos casos se han formado por embalsado natural de escorrentía, a causa de médanos, montículos loésicos o cordones de conchillas (Quirós, 2002). Tienen un origen geomorfológico común, con un perfil típico de Pfanne o Wanne (Ringuelet et al., 1965). La profundidad media y máxima varía de acuerdo a la superficie inundada. El drenaje, especialmente de las más pequeñas y la canalización creciente en busca de la utilización del humedal con fines agrícolas, está llevando a que su abundancia y tamaño disminuya constantemente. Carecen de estratificación térmica y por lo tanto poseen una circulación continua todo el año. Son naturalmente eutróficas e hipertróficas y actual- mente se encuentran bajo estrés ambiental ocasionado por el alto contenido de nutrientes en el entorno (Quirós et al., 2002). La gran mayoría presenta una hidrografía altamente variable dependiente de las precipitaciones in situ, con salinidades muy diversas, las más salinas se sitúan en cuencas hidrográficas más aisladas (Quirós y Drago, 1999; Quirós, et al., 2002). Su concentración salina permite clasificarlas como lagos subsalinos y salinos y en algunos casos como lagos de agua dulce, estos últimos dependientes de las precipitaciones que se producen en otoño (Ringuelet et al., 1967; Vervoost, 1967; Fuschini Mejía, 1994). Los suelos presentan concentraciones importantes de nutrientes lo que se ve reflejado en la alta productividad de sus aguas (Quirós et al., 2002).

Uno de los componentes principales de las comunidades biológicas de los sistemas acuáticos es el zooplancton. Este representa el eslabón entre el fitoplancton y los consumidores secundarios (Conde-Porcuna et al., 2004). El estudio cualitativo brinda información sobre las condiciones generales del ambiente y la funcionalidad de la comunidad. Por otro lado, los datos cuantitativos reflejan el grado de productividad del cuerpo de agua y permiten analizar el estado trófico caracterizándolos en cuanto a la calidad y aprovechamiento de sus aguas (Quirós, 1991; Boveri y Quirós, 2002; Quirós et al., 2002; Rennella y Quirós, 2002; Sosnovsky y Quirós, 2003; Rennella y Quirós, 2006). Los conocimientos de la biodiversidad y la biogeografía de lagunas de Sudamérica son escasos o fragmentarios. El zoo- 
plancton de los cuerpos de agua de la región de la llanura chaco-pampeana se caracteriza por la presencia de rotíferos, cladóceros y copépodos. Se conoce la composición del zooplancton sudamericano principalmente en las cuencas de los grandes ríos: Amazonas, Orinoco y del Plata y lagunas costeras de Río de Janeiro, etc. (Arcifa et al., 1992; Bozelli, 1994; Lewis et al., 1995; Junk, 1997; Arcifa et al., 1998). En Argentina, en la cuenca del Paraná se ha estudiado la abundancia y biodiversidad de microcrustáceos, identificándose diversos géneros de cladóceros y copépodos. Se destacan Alona, Bosmina, Moina, Daphnia, Ceriodaphnia y Boeckella, Notodiaptomus, Acanthocyclops, Methacyclops, Microcyclops, respectivamente (Paggi y José de Paggi, 1990; Paggi, 1993; José de Paggi y Paggi, 1998). También se ha estudiado la taxonomía, la distribución geográfica, determinación de biomasa zooplanctónica y factores que afectan la ecología de los diferentes cuerpos de agua (Pecorari et al., 2006; José de Paggi y Paggi, 2007). En la región de la llanura pampeana los estudios han estado restringidos a la pampa oriental húmeda, particularmente a las provincias de Buenos Aires y La Pampa. En ellos se dan a conocer la composición específica y riqueza del zooplancton y su relación con las distintas variables ambientales (Echaniz y Vignatti, 1996; Pilati, 1997; Vignatti, et al. 2007). Se identificaron especies de rotíferos cosmopolitas principalmente del género Brachionus, así como especies de cladóceros y copépodos endémicas para la región Neotropical, destacándose los géneros Moina, Daphnia, Boeckella, Cletocamptus, entre otros (Quirós, 1998; Pilati, 1999; Echaniz y Vignatti, 2002; Vignatti, et al. 2007). En su mayoría, estas pequeñas lagunas son de origen endorreico, muchas de las cuales poseen una composición salina elevada ( 8.4 a 20.8 g/l) con profundidades no mayores a $2.5 \mathrm{~m}$. En ellas se destaca la dominancia de los iones sodio y cloruro y de acuerdo a su pH son alcalinas (entre 9 y 11). La transparencia del agua es muy variable respecto de la profundidad (Quirós, 1998; Pilati, 1999; Echaniz y Vignatti, 2002; Quirós et al., 2002; Rennella y Quirós, 2002; Echaniz et al., 2005; Echaniz et al., 2006; Rennella y Quirós, 2006).
En el Sudoeste de la provincia de Buenos Aires sólo existen datos de calidad y aprovechamiento de las aguas de la Laguna Unamuno (Bohn et al., 2004), no existiendo información hasta la actualidad de la Laguna Calderón. El objetivo de este estudio es conocer las condiciones limnológicas de dos lagunas de la región pampeana y brindar información sobre los microcrustáceos planctónicos que habitan en estos ecosistemas.

\section{ÁREA DE ESTUDIO}

Las lagunas estudiadas se ubican en la región semiárida del sudoeste de la provincia de Buenos Aires, Argentina. La Laguna Unamuno $\left(38^{\circ} 54^{\prime} 10.9^{\prime \prime}\right.$ S- $61^{\circ} 51^{\prime} 37.4^{\prime \prime}$ W) se localiza en la cuenca del Arroyo Napostá Chico $\left(1320 \mathrm{~km}^{2}\right)$, ocupa una superficie de $3.96 \mathrm{~km}^{2}$, recibiendo un aporte de $0.8 \mathrm{~m}^{3} / \mathrm{seg}$. y posee una profundidad máxima de 3.5 m (Melo, 1995; Bohn, 2003). La Laguna Calderón ( $38^{\circ} 43^{\prime} 27.9^{\prime \prime}$ S-62 $\left.2^{\circ} 26.1^{\prime \prime} \mathrm{W}\right)$ se encuentra separada de la Laguna Unamuno por una distancia de $34 \mathrm{~km}$, posee una profundidad máxima de $0.7 \mathrm{~m}$ y cubre una superficie de $1.72 \mathrm{~km}^{2}$ (Fig. 1). Estos dos cuerpos de agua de

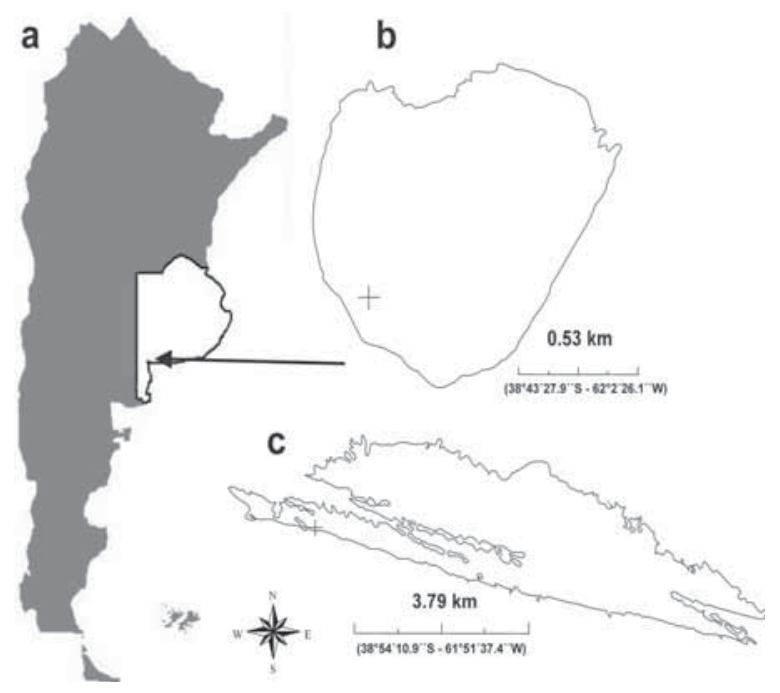

Figura 1. Localización del área de estudio. (a) Provincia de Buenos Aires en Argentina, (b) Laguna Calderón, (c) Laguna Unamuno. Study area location. (a) Buenos Aires Province in Argentina, (b) Calderón shallow pond, (c) Unamuno shallow pond. 
origen endorreicos se desarrollan en una planicie que no es uniforme y las aguas discurren de norte a sur. En sus márgenes se observan costras salinas y áreas de suelo desnudo, advirtiéndose praderas halófilas sin otras especies que colonicen el ambiente. La zona se caracteriza por poseer un clima templado, con valores medios anuales de temperatura comprendidos entre 14 y $20^{\circ} \mathrm{C}$ $\mathrm{y}$ con estaciones térmicas bien diferenciadas. El viento predomina de los cuadrantes NNW durante todo el año con una velocidad media anual de $22.5 \mathrm{~km} / \mathrm{h}$. Las lluvias no son suficientes para el cultivo y otorgan un carácter subhúmedo a este tipo de clima. Las precipitaciones presentan valores máximos en marzo $(90.9 \mathrm{~mm})$ y en octubre $(80.9 \mathrm{~mm}$ ) (Capelli de Steffens y Campo de Ferreras, 1994; Bohn et al., 2004).

\section{MATERIAL Y MÉTODOS}

El estudio se llevó a cabo durante la estación de primavera, donde se manifiestan las mayores abundancias del zooplancton. Las muestras fueron tomadas en un sitio de la zona litoral con una periodicidad quincenal a partir del 06 de Octubre al 21 de Diciembre de 2006. Las variables fisicoquímicas como temperatura del agua, salinidad, conductividad eléctrica, $\mathrm{pH}$, turbidez y oxígeno disuelto, fueron medidas con una sonda Horiba U-10 y U-23. Las mediciones de temperatura se efectuaron en horas del mediodía (10-13 hs.). Los valores de temperatura y salinidad se registraron en la columna de agua en superficie, medio y fondo para la Laguna Unamuno y solo en superficie para la Laguna Calderón debido a su escasa profundidad. Para la medición de nutrientes, iones disueltos, clorofila y sólidos en suspensión se extrajo una muestra de agua con botella Van Dorn. La transparencia del agua se midió con disco Secchi. Se tomaron muestras del fondo de cada laguna para el análisis de la textura del suelo. Las variables meteorológicas fueron obtenidas de una estación cercana Weather Monitor II.

El zooplancton se obtuvo con red cónica de $150 \mu \mathrm{m}$ de apertura de malla. Los muestreos cuantitativos se efectuaron por duplicado mediante arrastres verticales desde la proximidad
Tabla 1. Parámetros físicos para ambas lagunas. Media (X), desvío estándar (SD), máximo (máx.) y mínimo (min.). Physical parameters for both lagoons. Average (x), standard deviation (SD), maximum (max) and minimum (min).

\begin{tabular}{crrrr}
\hline & \multicolumn{2}{c}{ Laguna Unamuno } & \multicolumn{2}{c}{ Laguna Calderón } \\
\cline { 2 - 5 } & \multicolumn{1}{c}{$\mathrm{X}$} & máx. & \multicolumn{1}{c}{ X } & \multicolumn{1}{c}{ máx. } \\
& $\mathrm{SD}$ & \multicolumn{1}{c}{ min. } & \multicolumn{1}{c}{$\mathrm{SD}$} & \multicolumn{1}{c}{ min. } \\
\hline Temperatura & 19.36 & 23.20 & 17.42 & 26.00 \\
$\left({ }^{\circ} \mathrm{C}\right)$ & 2.66 & 16.30 & 5.44 & 11.20 \\
\hline Salinidad & 0.39 & 0.43 & 1.69 & 2.14 \\
$(\mathrm{~g} / \mathrm{l})$ & 0.03 & 0.36 & 0.37 & 1.29 \\
\hline Conductividad & 7232.00 & 7870.00 & 27680.00 & 34500.00 \\
$(\mu \mathrm{S} / \mathrm{cm})$ & 484.17 & 6770.00 & 5418.67 & 21500.00 \\
\hline $\mathrm{pH}$ & 9.56 & 9.94 & 10.17 & 10.27 \\
& 0.36 & 9.14 & 0.10 & 10.06 \\
\hline Sedimento & 66.40 & 155.00 & 462.00 & 605.00 \\
$(\mathrm{mg} / \mathrm{l})$ & 51.07 & 30.00 & 87.66 & 385.00 \\
\hline Transparencia & 0.28 & 0.45 & 0.11 & 0.17 \\
$(\mathrm{~m})$ & 0.14 & 0.06 & 0.07 & 0.05 \\
\hline Oxígeno disuelto & 8.91 & 10.45 & 10.20 & 13.54 \\
$(\mathrm{mg} / \mathrm{l})$ & 1.14 & 7.46 & 2.57 & 6.42 \\
\hline Clorofila “ $a$ " & 94.76 & 275.56 & 19.55 & 75.54 \\
$(\mu \mathrm{g} / \mathrm{l})$ & 106.83 & 2.63 & 31.36 & 2.90 \\
\hline Feopigmentos & 22.38 & 65.43 & 12.48 & 29.98 \\
$(\mu \mathrm{g} / \mathrm{l})$ & 24.50 & 8.72 & 11.84 & 2.29 \\
\hline Turbidez & 66.40 & 155.00 & 462.40 & 605.00 \\
$(\mathrm{NTU})$ & 51.07 & 30.00 & 87.66 & 385.00 \\
\hline & & & &
\end{tabular}

del fondo hacia la superficie obteniéndose un volumen medio de $12 \times 10^{2}$ litros. Las muestras se fijaron con formaldehído al $4 \%$. Para identificar organismos más pequeños, se colectaron muestras con botella Van Dorn de 2.5 1. Estas se fijaron en recipientes de $250 \mathrm{ml}$ con lugol, en proporción $0.5 \mathrm{ml} / 100 \mathrm{ml}$. Los nutrientes se determinaron mediante las siguientes técnicas: fosfatos, técnica de Eberlein y Kattner (1987); nitratos, Grasshof (1969); nitritos, Grasshof et al. (1983) y silicatos, mediante el uso del autoanalizador Technicon II, método 186-72 W/B. La clorofila y feopigmentos se determinaron por el método de Lorenzen (1967) La clorofila $a$ no fue corregida por feopigmentos. Los cationes y aniones presentes en el agua se determinaron mediante la técnica APHA-AWWA-WPCF (1992). Las muestras se llevaron a un volumen de $1000 \mathrm{ml}$ para el conteo directo, en los casos donde el zooplancton era abundante se analizaron 2 alícuotas de $20 \mathrm{ml}$. Los 
organismos fueron identificados bajo microscopio binocular convencional (Olympus) y microscopio estereoscópico (Olympus), para el conteo se utilizó una cámara tipo Sedgwick-Rafter. La abundancia fue expresada en ind/l. De los taxones más abundantes se midió longitud y ancho máximo en 100 ejemplares. Para identificar organismos más pequeños se utilizó la técnica de sedimentación a partir de las muestras de botella Van Dorn. Se calculó la diversidad específica mediante el Índice de Diversidad H de Shannon" (Shannon y Weaver, 1949) y la equitatividad y el Índice de Dominancia Mc. Naughton (1968). La asociación estadística entre variables se realizó por medio del coeficiente de correlación de Pearson. El análisis de los datos fisicoquímicos se llevó a cabo mediante el análisis de componentes principales (ACP). Los datos de la matriz fueron transformados como $\operatorname{Ln}(x+1)$.

\section{RESULTADOS}

En la Laguna Unamuno la temperatura en la columna de agua no evidenció estratificación térmica vertical. En la Laguna Calderón tampoco se evidenció este fenómeno, debido a su escasa profundidad. La temperatura superficial del agua se correlacionó con la temperatura del aire de forma significativa (Unamuno, $r=0.68, p<0.01$, $n=5$; Calderón de $r=0.79, p<0.01, n=5$ ). En relación a la salinidad la Laguna Unamuno no presentó estratificación y su concentración media fue menor con respecto a la Laguna Calderón. La conductividad presentó valores más altos en la Laguna Calderón que en la Unamuno. $\mathrm{El} \mathrm{pH}$ fue alcalino en los dos cuerpos de agua, fluctuando entre 9 a 10. La presencia de sólidos disueltos se correspondió con la turbidez (Unamuno $r=0.95, p<0.10$; Calderón, $r=0.95$, $p<0.05)$. El sedimento del fondo estuvo representado en la Laguna Unamuno por un $91.57 \%$ de fracción gruesa (arena y grava) y $8.43 \%$ de fracción fina (limo y arcilla), constituyendo un suelo de tipo arenoso franco. En la Laguna Calderón el porcentaje de fracción gruesa fue inferior $(66.51 \%)$ y se observó un aumento sustancial de la fracción fina $(33.49 \%)$, representando
Tabla 2. Concentraciones de nutrientes. Media (X) y desvío estándar (SD). Nutrient concentrations. Average $(X)$ and standard deviation $(S D)$.

\begin{tabular}{ccccr}
\hline \multirow{2}{*}{$\begin{array}{c}\text { Nutrientes } \\
(\mathrm{mg} / \mathrm{l})\end{array}$} & \multicolumn{2}{c}{ Laguna Unamuno } & \multicolumn{2}{c}{ Laguna Calderón } \\
\cline { 2 - 5 } & $\mathrm{X}$ & \multicolumn{1}{c}{$\mathrm{SD}$} & \multicolumn{1}{c}{$\mathrm{X}$} & $\mathrm{SD}$ \\
\hline Nitratos & 0.77 & 0.59 & 0.44 & 0.22 \\
Nitritos & 0.30 & 0.21 & 0.18 & 0.20 \\
Fosfatos & 4.23 & 1.85 & 11.58 & 9.05 \\
Silicatos & 9.07 & 14.12 & 32.72 & 53.35 \\
Amonio $(\mu \mathrm{M} / \mathrm{l})$ & 52.36 & 40.71 & 42.03 & 28.17 \\
\hline
\end{tabular}

un suelo de tipo franco arcillo arenoso. La clorofila $a$ fue altamente variable para la Laguna Unamuno, fluctuando de $2.63 \mu \mathrm{g} / \mathrm{l}$ en octubre hasta llegar a valores de $275.56 \mu \mathrm{g} / \mathrm{l}$ en diciembre $(\mathrm{X}=94.76, \mathrm{SD}=95.56)$. En Laguna Calderón estos valores fueron mucho más bajos, del orden de $2.90 \mu \mathrm{g} / \mathrm{l}$ en noviembre y $75.54 \mu \mathrm{g} / \mathrm{l}$ en diciembre $(\mathrm{X}=19.55, \mathrm{SD}=28.05 \mu \mathrm{g} / \mathrm{l})($ Tabla 1$)$.

En la Laguna Unamuno el valor medio de los nitratos osciló entre $0.06 \mathrm{mg} / \mathrm{l}$ y $1.69 \mathrm{mg} / \mathrm{l}$, las concentraciones de nitritos fueron altas, oscilando entre 0.03 y $0.50 \mathrm{mg} / \mathrm{l}$. Los silicatos fluctuaron entre 0.85 y $34.20 \mathrm{mg} / \mathrm{l}$. Se obtuvieron concentraciones de fosfatos del orden de $1.47 \mathrm{mg} / \mathrm{l} \mathrm{a}$ $6.68 \mathrm{mg} / 1$. En la Laguna Calderón las concentraciones de nitratos (entre $0.10 \mathrm{mg} / \mathrm{l}$ y $0.57 \mathrm{mg} / \mathrm{l}$ ) y nitritos (entre $0.01 \mathrm{mg} / \mathrm{l}$ y $0.50 \mathrm{mg} / \mathrm{l}$ ) fueron inferiores a las registradas en la Laguna Unamuno. Inversamente, los silicatos (entre $2.13 \mathrm{mg} / \mathrm{l}$ y $127.64 \mathrm{mg} / \mathrm{l}$ ) y los fosfatos (entre $3.30 \mathrm{mg} / \mathrm{l}$ y $21.76 \mathrm{mg} / \mathrm{l}$ ) presentaron concentraciones superiores (Tabla 2).

Del ACP se desprende que ambas lagunas presentaron similar composición iónica (Fig. 2a, b). Las concentraciones de cationes se ordenaron de la siguiente manera: $\mathrm{Na}>\mathrm{K}>\mathrm{Mg}>\mathrm{Ca}$; siendo la concentración del sodio muy superior en Laguna Calderón. Los aniones se ordenaron de la siguiente manera: en la Laguna Unamuno $\mathrm{Cl}>\mathrm{CO}_{3}>\mathrm{SO}_{4}>\mathrm{HCO}_{3}$ y en la Laguna Calderón $\mathrm{CO}_{3}>\mathrm{Cl}>\mathrm{SO}_{4}>\mathrm{HCO}_{3}$. Aunque los cloruros fueron dominantes en las dos lagunas, los sulfatos representaron el 21 (Unamuno) y $25 \%$ (Calderón). De acuerdo a las concentraciones de $\mathrm{HCO}_{3}$, la Laguna Unamuno presentó una alcalinidad más baja que la Laguna Calderón (Tabla 3).

A partir del ACP se caracterizó a las lagunas teniendo en cuenta los principales parámetros 

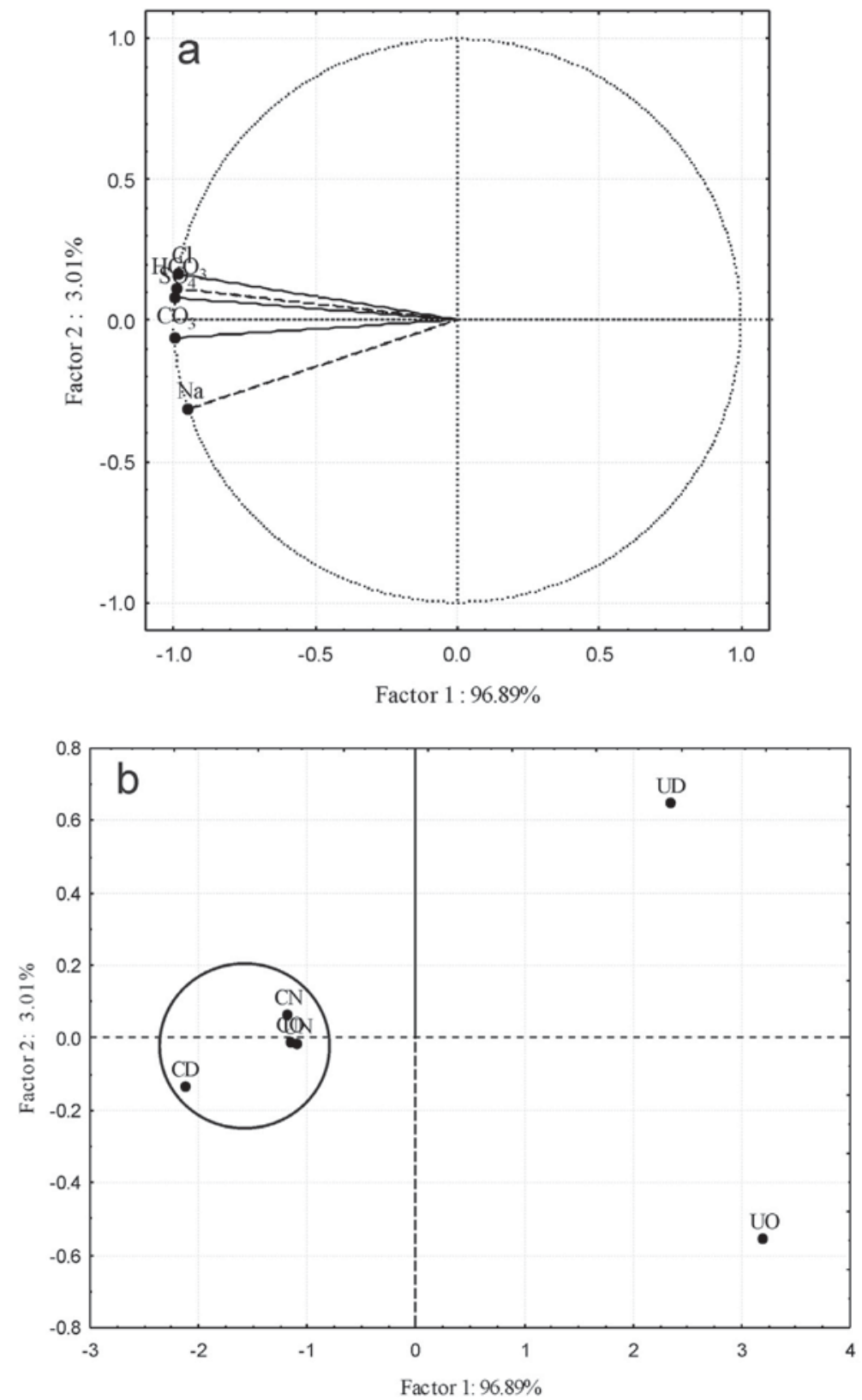

Figura 2. Análisis de componentes principales de la composición iónica para la Laguna Unamuno y Laguna Calderón. (a) Distribución de iones (b) Disposición de las fechas de muestreo en relación a los dos primeros ejes. Cl: cloro, $\mathrm{HCO}_{3}$ : bicarbonatos, $\mathrm{SO}_{4}$ : sulfatos, $\mathrm{CO}_{3}$ : carbonatos, Na: sodio. U: Unamuno, C: Calderón, O: octubre, N: noviembre, D: diciembre. Principal component analysis of the ionic composition for Unamuno and Calderon lagoons. (a) Ions distribution (b) Sampling dates distribution in relation to the two first axes. $\mathrm{Cl}$ : chloride, $\mathrm{HCO}_{3}$ : bicarbonates, $\mathrm{SO}_{4}$ : sulphates, $\mathrm{CO}_{3}$ : carbonates, $\mathrm{Na}$ : sodium. U: Unamuno, C: Calderon, O: october, $N$ : november, D: december.

abióticos. Los dos primeros ejes contribuyeron a explicar el $78.67 \%$ de la variabilidad de los datos. Las variables que formaron parte del primer eje $(52.90 \%)$ fueron la turbidez, conductividad, materia orgánica particulada, profundidad y los iones nitritos y silicatos (Fig. 3a, b). Según estas 
variables los puntos que representaron a la Laguna Unamuno, se ubicaron sobre el primer eje en el sector izquierdo, entre los cuadrantes tres y cuatro, ya que ésta laguna presentó una mayor profundidad, valores más altos de nitritos y bajas concentraciones de silicatos. En tanto que
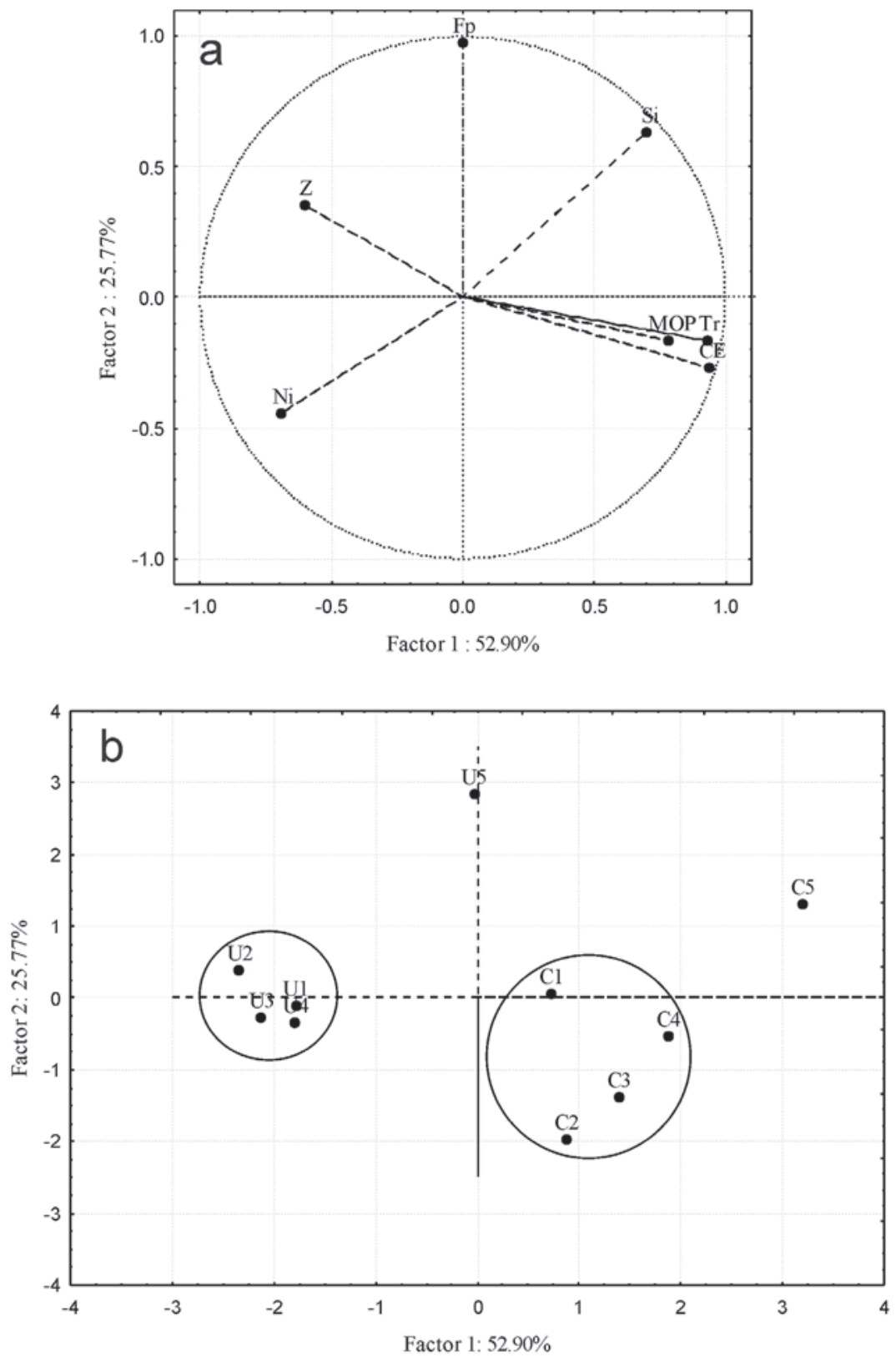

Figura 3. Análisis de componentes principales para variables ambientales en la Laguna Unamuno y Laguna Calderón. (a) Distribución de las variables (b) Disposición de las fechas de muestreo en relación a los dos primeros ejes. Z: profundidad, Fp: feopigmentos, Si: silicatos, Ni: nitritos, MOP: materia orgánica particulada, Tr: turbidez, CE: conductividad eléctrica. U: Unamuno, C: Calderón, 1: 06/10/06, 2: 02/11/06, 3: 20/11/06, 4: 11/12/06, 5: 21/12/06. Principal component analysis for environmental variables in the Unamuno and Calderon lagoons. (a) Variables distribution (b) Sampling dates distribution in relation to the two first axes. Z: depth, Fp: feopigments, Si: silicates, Ni: nitrites, MOP: particulate organic material, Tr: turbidity, CE: electric conductivity. U: Unamuno, C: Calderon, 1: 10/06/06, 2: 11/02/06, 3: 11/20/06, 4: 12/11/06, 5: 12/21/06. 
Tabla 3. Concentraciones de cationes y aniones. Media (X) y desvío estándar (SD). Cation and anion concentrations. Average $(X)$ and standard deviations $(S D)$.

\begin{tabular}{crrrr}
\hline Iones & \multicolumn{2}{c}{ Laguna Unamuno } & \multicolumn{2}{c}{ Laguna Calderón } \\
\cline { 2 - 5 }$(\mathrm{mg} / \mathrm{l})$ & \multicolumn{1}{c}{$\mathrm{X}$} & \multicolumn{1}{c}{$\mathrm{SD}$} & \multicolumn{1}{c}{$\mathrm{X}$} & \multicolumn{1}{c}{$\mathrm{SD}$} \\
\hline $\mathrm{Ca}^{++}$ & 17.25 & 9.18 & 8.91 & 7.41 \\
$\mathrm{Mg}^{++}$ & 28.20 & 13.12 & 21.30 & 2.84 \\
$\mathrm{Na}^{+}$ & 3850.00 & 2530.00 & 7607.33 & 1607.08 \\
$\mathrm{~K}^{+}$ & 52.82 & 15.43 & 60.09 & 16.70 \\
$\mathrm{HCO}_{3}^{-}$ & 569.33 & 712.63 & 1846.67 & 444.67 \\
$\mathrm{CO}_{3}^{=}$ & 3053.33 & 2305.39 & 6580.00 & 1195.00 \\
$\mathrm{Cl}^{-}$ & 3278.00 & 2068.00 & 6272.00 & 1118.00 \\
$\mathrm{SO}_{4}^{=}$ & 1811.00 & 2017.00 & 4838.33 & 1027.62 \\
\hline
\end{tabular}

sobre el segundo cuadrante se ubicaron aquellos puntos que representan a la Laguna Calderón, la cual mostró una baja profundidad y altos valores de conductividad eléctrica, turbidez, materia orgánica particulada y silicatos. El segundo eje $(25.77 \%)$ estuvo explicado principalmente por los feopigmentos, debido a esto, la muestra del 21/11 para Laguna Unamuno se alejó de los demás puntos como consecuencia de la elevada concentración que presentó para el pigmento, lo mismo sucedió para la muestra del 21/11 de la Laguna Calderón. En relación a las variables meteorológicas, la velocidad media del viento fue $28.7 \mathrm{~km} / \mathrm{h}$ en la Laguna Unamuno (min. = $13-$ máx. $=47 \mathrm{~km} / \mathrm{h})$ y $24.4 \mathrm{~km} / \mathrm{h}$ en la Laguna Calderón $(\min .=7-$ máx. $=48 \mathrm{~km} / \mathrm{h})$. La dirección predominante fue del NNW. La precipitación anual acumulada fue de $388.2 \mathrm{~mm}$. Las máximas precipitaciones anuales fueron durante el mes de octubre (media mensual de $105.2 \mathrm{~mm}$ ), en tanto que en noviembre las precipitaciones alcanzaron una media de $30 \mathrm{~mm}$ y en diciembre de $45 \mathrm{~mm}$. La humedad relativa fue del $70 \%$.

El zooplancton estuvo representado por 6 especies de microcrustáceos. En ambos cuerpos de agua se evidenció ausencia de rotíferos. Se hallaron elementos ticoplanctontes, como ostrácodos, anfípodos juveniles del género Hyalella curviseriata y $H$. pampeana, larvas de insectos dípteros y escasas larvas y adultos de poliquetos. No existen evidencias de la presencia de peces para ambos cuerpos de agua (Tabla 4). En la Laguna Unamuno la densidad del zooplancton fluctuó entre 221 y 1703 ind/l. La especie predominante fue $M$. eugeniae. La mayor abundancia se registró durante el mes de diciembre con 1703 ind/l (Fig. 4a). En la Laguna Calderón el zooplancton fluctuó entre 41 y 1401 ind/l, siendo la especie dominante $B$. poopoensis, le siguió en importancia $M$. eugeniae. La mayor abundancia se registró a fines de noviembre (Fig. 4b). La diversidad específica promedio en la Laguna Unamuno fue de 2.69 bits/ind y la equitatividad de 0.96. Para la Laguna Calderón se obtuvo una diversidad de 0.926 bits/ind y una equitatividad de 0.6. El valor del "Índice de Dominancia" fue de $98 \%$ para la Laguna Unamuno y de $99 \%$ para la Laguna Calderón. M. eugeniae presentó una longitud de $1.3 \mathrm{~mm}$ (mín. $=1.1-$ máx. $=1.5 \mathrm{~mm}$ ) y un ancho de $1 \mathrm{~mm}$ (mín. = 0.9- máx. = $1.2 \mathrm{~mm}$ )

Tabla 4. Especies del zooplancton en las lagunas Unamuno y Calderón y su porcentaje de ocurrencia. $O$ : observado, $N O:$ no observado. Zooplankton species in the Unamuno and Calderon lagoons and their occurrence percentage. O: observed, NO: not observed.

\begin{tabular}{lcc}
\hline Taxones determinados & Laguna Unamuno (\%) & Laguna Calderón (\%) \\
\hline Ciliophora & & $N O$ \\
Strombidium sp. (Claparede \& Lachmann, 1858) & & \\
\hline Cladocera & 70 & 34 \\
Moina eugeniae (Olivier, 1954) & 0.2 & $N O$ \\
Alona diaphana (King, 1853) & & 66 \\
Copepoda & 27 & $N O$ \\
Boeckella poopoensis (Marsh, 1906) & 1.2 & $N O$ \\
Metacyclops mendocinus (Wierzejski, 1892) & 0.2 & $N O$ \\
Microcyclops anceps (Richard, 1897) & 1.4 & $N O$ \\
Cletocamptus deitersi (Richard, 1897) & $O$ & \\
larva nauplii & &
\end{tabular}



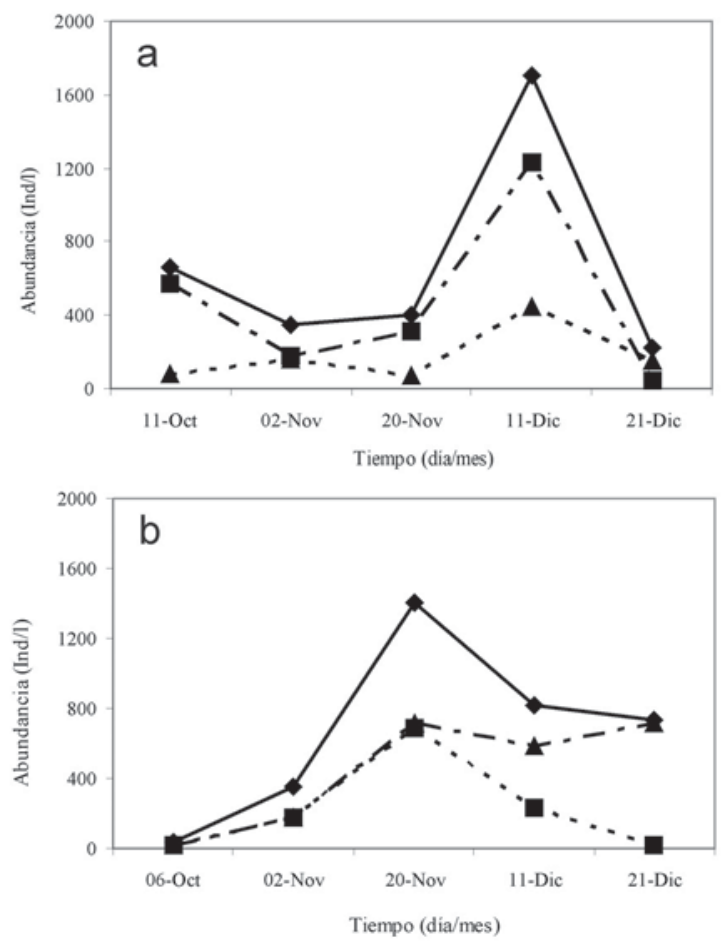

Figura 4. Variación de la abundancia del zooplancton en las lagunas Unamuno (a) y Calderón (b). Abundancia total (\$), abundancia de $M$. eugeniae (ם) y abundancia de B. poopoensis $(\mathbf{\Delta})$. Variation of zooplankton abundance in the Unamuno (a) and Calderón lagoons $(b)$. Total abundance $(\boldsymbol{\nabla})$, M. eugeniae abundance (-) and $\mathrm{B}$. poopoensis abundance $(\mathbf{\Delta})$.

y B. poopoensis registró un largo de $1.56 \mathrm{~mm}$ $(\min .=0.8-$ máx. $=2.3 \mathrm{~mm})$ y un ancho de $0.4 \mathrm{~mm}(\mathrm{~min} .=0.3-$ máx. $=0.6 \mathrm{~mm})$.

\section{DISCUSIÓN}

El zooplancton estuvo representado por dos microcrustáceos, M. eugeniae y A. diaphana y el copépodo endémico $B$. poopoensis. M. eugeniae se encuentra entre las especies más citadas en los registros del zooplancton de la región, conformando una asociación zooplanctónica típica junto a Daphnia menucoensis (Ringuelet et al., 1965; Dipolito, 2006). Para la laguna pampeana Los Manantiales, de característica más salina que las lagunas del sudoeste bonaerense Echaniz et al. (2005, 2006) encontraron la misma asociación. En las lagunas objeto de estudio D. menucoensis, estuvo reemplazada por $B$. poopoensis. La presencia ocasional de $A$. diaphana en el plancton respondió al tipo de muestreo realizado, la misma es encontrada comúnmente en el fondo (Pennak, 1978; Meneses Junco, 1997). Cletocamptus deitersi, Metacyclops mendocinus y Microcyclops anceps son especies comunes para otras lagunas de la región con salinidades que fluctúan entre 8-20 g/l; estos copépodos son considerados endémicos para el área (Echaniz et al., 2005, 2006). La dominancia de especies endémicas halladas en este estudio es señalada también para el zooplancton de lagunas de Sudamérica, que difiere de aquellas de ambientes del hemisferio norte y continente australiano (Halse et al., 1998; Timms, 1998; Derry et al., 2003). Los rotíferos son considerados un grupo cosmopolita, sin embargo no fueron registrados durante el período de estudio a pesar de utilizar dos metodologías de muestreo (red y botella), los mismos presentarían pulsos poblacionales en otras épocas del año.

Los índices aplicados de diversidad, equidad y dominancia indican que, durante el período de primavera existió una fuerte dominancia de pocas especies sobre el total de individuos y una baja diversidad. La presencia de zoopláncteres de gran tamaño (cladóceros y copépodos) halladas en este estudio también es menciona por Quirós, et al. (2002) para la misma época del año. En Sudamérica a diferencia de los ambientes marinos, los ecosistemas de aguas continentales presentan un zooplancton generalmente dominado por animales de pequeño tamaño como los rotíferos, pequeños cladóceros y estadios inmaduros de copépodos. Por otro lado, la superioridad de zooplancton de gran tamaño es señalada como un factor supresor del crecimiento del fitoplancton (Conde-Porcuna et al., 2004). Brooks y Dodson (1965) estudiando cuerpos de agua de Nueva Inglaterra plantearon la hipótesis de "Eficiencia-Tamaño". Dichos autores postularon que los grandes zooplanctontes serían más eficientes en el pastoreo sobre la biomasa fitoplanctónica que sus competidores más pequeños, por lo que estos últimos estarían restringidos a depender de pequeñas partículas. Nuestros resultados hallados sustentarían esta hipótesis. Sumado a esto los peces selectivamente sólo depre- 
darían a los grandes zooplanctontes, constituyendo otro factor adicional que favorecería la presencia de un zooplancton de gran tamaño. El fitoplancton de la Laguna Unamuno está integrado principalmente por algas cyanophytas (70-90\%) y chlorophytas (3-10\%). Por el contrario en la Laguna Calderón predominaron las chlorophytas (70-90\%) y las diatomeas (4-10\%) (Andrade et al., 2007). Todas estas microalgas podrían servir de alimento a la alta densidad zooplanctónica.

Teniendo en cuenta los altos valores de abundancia hallados en este estudio se caracterizaron a las lagunas como eutróficas. Para lagunas saladas de la provincia de La Pampa, Echaniz et al. (2006) citan densidades del zooplancton altamente variables desde 26 a 80000 ind/l y Maizels et al. (2003) estudiando la laguna Chascomús (Prov. de Buenos Aires) hallaron densidades de 440 ind/l. Las variaciones en el número de individuos durante los meses de primavera responderían a fluctuaciones que se producen a lo largo del ciclo anual y a variaciones de la temperatura del agua. En la Laguna Unamuno la densidad máxima coincidió con el pico de máxima temperatura, en promedio la más elevada de todo el muestreo ya que dicho período fue el más cercano a la estación estival $\left(23^{\circ} \mathrm{C}-11\right.$ de diciembre). Para la Laguna Calderón este mismo comportamiento se produjo 20 días antes. Los bajos valores de abundancia de M. eugeniae registrados para ambas lagunas, a fines de diciembre podrían estar relacionados con la disminución de la temperatura del agua $\left(3^{\circ} \mathrm{C}\right)$. Además de la temperatura, otros factores que se podrían plantear para tratar de explicar la muy baja abundancia el 21/12 serían factores tanto físicos como biológicos, predación por invertebrados, sobrepastoreo del propio plancton y distribución en parches del plancton originada por el viento.

Los microcrustáceos hallados en este estudio fueron representativos de lagunas oligohalinas (cladóceros, copépodos y escasez absoluta del género Daphnia). Por otra parte, la existencia de poblaciones de boeckellidos es un rasgo diferente a lo que comúnmente es señalado en este tipo de lagunas, por ello en este sentido se asemejaría más al plancton de aguas mesohalinas (bajo número y variedad de rotíferos y microcrustáceos) (Acosta et al., 2003). La mayor abundancia de B. poopoensis en la Laguna Calderón podría explicarse a que ésta se halla presente a salinidades altas $(80 \mathrm{~g} / \mathrm{l})$, con predominio de iones cloruro (Bayly, 1992). Estas dos características fueron observadas en la Laguna Calderón. La presencia de elementos adventicios o ticoplanctontes como ostrácodos y cladóceros bentónicos (Alona sp.) podría estar relacionada a la ausencia o escasez de vegetación fanerógama emergente o sumergida que le sirva de hábitat para arraigarse (Ringuelet, 1972).

En términos fisicoquímicos las lagunas fueron clasificadas como oligohalinas según Ringuelet (1972). Bohn et al. (2004) señalan para la Laguna Unamuno que es más conveniente caracterizarla en términos de conductividad eléctrica y no de salinidad, dichos autores hallaron valores inferiores a los registrados en este estudio. Las concentraciones de nutrientes fueron superiores a las obtenidas por dichos autores y por encima de los considerados característicos en el caso de nitritos y silicatos. En la Laguna Unamuno los nitratos se hallaron dentro del rango de valores bajos no así los silicatos, los cuales superaron ampliamente los valores considerados característicos de lagunas de zonas templadas (0 a $5 \mathrm{mg} / \mathrm{l}$ ). Los nitritos fueron más altos que los encontrados por Bohn et al. (2004) y estuvieron por encima del límite que se considera aceptable $(0.07 \mathrm{mg} / \mathrm{l}$.), Nixon (1981) estudiando 39 lagunas en Méjico consideró sólo a 3 de estos cuerpos de agua con valores elevados de nitritos. Los fosfatos también superaron ampliamente los valores considerados medios para lagunas de esta región (Bohn et al. (2004). Las altas concentraciones de fosfatos halladas en este estudio responderían a la baja solubilidad que tiene este elemento y a la característica endorreica de la cuenca (Quirós, 1991); lamentablemente hay escasos datos para lagunas de la provincia de Buenos Aires que estén disponibles para correlacionar. Para la Laguna Calderón, los nitritos, fosfatos y silicatos, estuvieron por encima de los valores considerados característicos para este tipo de cuerpos de agua. Los mismos, fueron coincidentes a los hallados por Geraldi (2003) en la laguna Malaver, muy próxima a la Laguna Calderón. La presencia de diatomeas en 
esta laguna sería la responsable de los elevados valores de sílice. Las concentraciones de amonio en los dos cuerpos de agua estuvieron dentro del rango de concentraciones medias (Nixon, 1981).

En base a la concentración de iones podemos clasificar a las lagunas como sódicas bicarbonatadas cloruradas (Ringuelet et al. 1967). Siguiendo el criterio de Ringuelet et al. (1965), que señala la relación $\mathrm{Mg}^{+} / \mathrm{Ca}$ y $\mathrm{Ca}+\mathrm{Mg} / \mathrm{Na}+\mathrm{K}$ fluctúan a lo largo del año, la composición iónica fue característica de la estación de primavera. El marcado distanciamiento de las muestras de octubre y diciembre de la Laguna Unamuno en el ACP se correspondió con las bajas concentraciones medidas de los iones $\mathrm{Na}, \mathrm{HCO}_{3}, \mathrm{CO}_{3}, \mathrm{Cl}$ y $\mathrm{SO}_{4}$. Esto se debió al aporte significativo del arroyo Napostá Chico durante las altas precipitaciones registradas en el mes octubre y diciembre. La Laguna Calderón no presentaría estas características de dilución acentuada debido a que la misma sólo recibe aportes de las precipitaciones. Como es planteado por Quirós et al. (2002), en las lagunas pampeanas la cubeta es pequeña en relación al espejo de agua, lo que conduce a que el tiempo de permanencia del agua varíe directamente con el balance entre la precipitación y la evapotranspiración durante el ciclo de sequía-inundación característico de esta zona. Así, los casos de extrema sequía o intensas precipitaciones generan cambios importantes en la estructura y funcionamiento de una laguna pampeana típica. A su vez este efecto de concentración y dilución se ve reflejado en la abundancia de las comunidades bióticas. Sin embargo, las pequeñas lagunas pampeanas al poseer una área de drenaje mucho menor conlleva a que la variación de la concentración de sales sea menos drástica que en las más grandes. La clorofila estuvo por encima de valores medios propuestos para lagunas pampeanas (Quirós et al., 2002), la alta concentración de estos pigmentos podría constituir un buen indicador de la biomasa fitoplanctónica, indicando posibles pulsos de la comunidad algal. Los valores altos de sólidos en suspensión en la Laguna Calderón fueron similares a los encontrados por Geraldi (2003) para la laguna Malaver, dicha autora mi- dió concentraciones que van de 460 a 900 mg/l. Estas altas concentraciones son apreciables cuando se observa in situ el cuerpo de agua, con una coloración rojiza amarronada. El incremento de sedimento en la columna de agua se correspondería con la escasa profundidad, de esta forma el oleaje en superficie generaría una resuspensión de sedimento que dependerá del tipo y tamaño de las partículas, las más livianas, que son en un alto porcentaje de tipo franco arcillosas, se movilizarán desde el fondo y al no encontrar sitios donde acumularse permanecen constantemente en la columna de agua (Scheffer, 1998). Como consecuencia de este fenómeno la transparencia del agua fue baja; el disco Secchi se visualizó a muy pocos centímetros de la superficie. La turbidez medida en ambos cuerpos de agua se puede extender a otras lagunas de la región. Las teorías más actuales solo predicen que existen dos tipos de lagunas, separándolas en turbias y claras (Moss, 1990; Jeppesen et al., 1990; Scheffer, 1990; Scheffer et al., 1993). Teniendo en cuenta los resultados aquí obtenidos y los de otros autores para lagunas pampeanas y lagos de llanura poco profundos repartidos mundialmente, estos dos cuerpos de agua entrarían dentro del tipo “dos" de la clasificación de Quirós, et al. (2002) y Jeppesen, et al. (1991). Respondería a lagunas turbias, con baja transparencia y abundante desarrollo de fitoplancton, pero escasa presencia de macrófitas. Generalmente, presentan altas abundancias de peces planctívoros visuales, situación que no se manifestó durante el período de estudio. En base a las condiciones limnológicas y biológicas se caracterizaron a estas dos lagunas de la Región Pampeana. Las características fisicoquímicas pueden explicar la gran variabilidad del zooplancton y tendrían un efecto importante sobre la ecología de estos sistemas lagunares.

\section{AGRADECIMIENTOS}

Este trabajo fue realizado con el apoyo de los proyectos financiados por CONICET, Universidad Nacional del Sur y Agencia Nacional de Investigación Científica y Tecnológica. 


\section{REFERENCIAS}

ACOSTA, F., M. CADIMA \& M. MALDONADO. 2003. Patrones espaciales de la comunidad planctónica lacustre en un gradiente geofísico y bioclimático en Bolivia. Revista Boliviana de Ecología y Conservación Ambiental, 13: 31-53.

ANDRADE, G. M., N. FERRER, V. Y. BOHN \& M. C. PICCOLO. 2007. Estudio Preliminar del fitoplancton de la laguna Unamuno (Provincia de Buenos Aires). XXXI Jornadas Argentinas de Botánica, Corrientes, Argentina, 42: 193.

APHA. 1992. Standard Methods for the Examination of Water and Wastewater. $18^{\text {th }}$ ed. APHA (American Public Health Association), AWWA (American Water Works Association) \& WPCF (Water Pollution Control Federation). Washington, DC, USA. 1200 pp.

ARCIFA, M. S., E. A. T. GOMES \& A. J. MESCHIATTI. 1992. Composition and fluctuations of the zooplankton of tropical Brazilian reservoir. Arch. Hydrobiol., 123: 479-495.

ARCIFA, M. S., L. H. S. SILVA \& M. H. L. SILVA. 1998. The planktonic community in a tropical Brazilian reservoir: composition, fluctuations and interactions. Revista Brasileira de Biologia, 58: 241254.

BAYLY, I. A. E. 1992. The Non-Marine Centropagidae (Copepoda: Calanoida) of the world. In: Guides to the Identification of the Microinvertebrates of the Continental Waters of the World 2. Dumond, H. J. (coord.): 1-30. SPB Academic Publishing, The Hague, The Netherlands.

BOHN, V. Y. 2003. Calidad del agua en la laguna Unamuno. Provincia de Buenos Aires, Argenti$n a$. Tesis de Magíster Profesional en Levantamiento de Recursos Hídricos, Universidad Mayor de San Simón, Centro de Levantamientos Espaciales. Inédito. Cochabamba, Bolivia. $60 \mathrm{pp}$.

BOHN, V. Y., G. M. E. PERILLO \& M. C. PICCOLO. 2004. Calidad y aprovechamiento del agua de la laguna Unamuno (Buenos Aires, Argentina). $\mathrm{Pa}$ peles de Geografía, 40: 173-184.

BOVERI, M. B. \& R. QUIRÓS. 2002. Trophic interactions in Pampean shallow lakes: Evaluation of silverside cascading effects in mesocosm experiments. Verh. Internat. Verein. Limnol., 28: 12741278.

BOZELLI, R. L. 1994. Zooplankton community density in relation to water level fluctuations and inorganic turbidity in an Amazonian lake,
"Lago Batata", state of Para, Brazil. Amazoniana, 13(1-2): 17-32.

BROOKS, J. L. \& S. I. DODSON, 1965. Predation, body size, and composition of plankton. Science, 150: 28-35.

CAPELLI DE STEFFENS, A. \& A. CAMPO DE FERRERAS. 1994. La transición climática en el Sudoeste Bonaerense. Sigeo 5: Serie Monografías. Universidad Nacional del Sur. Bahía Blanca. Argentina. $76 \mathrm{pp}$.

CONDE-PORCUNA, J. M., E. RAMOS-RODRÍGUEZ \& R. MORALES-BAQUERO. 2004. El zooplancton como integrante en la estructura trófica de los ecosistemas lénticos. Ecosistemas, 2: 1-9.

DERRY, A. M. E., E. PREPAS \& P. D. N. HERBERT. 2003. A comparison of zooplankton communities in saline lake water with anion composition. Hydrobiologia, 505: 199-215.

DIPOLITO, A. 2006. Segundo registro de la especie Moina eugeniae Olivier, 1954 (Crustacea, Cladócera) en la provincia de Buenos Aires: Análisis morfológico comparativo. IV Congreso sobre Ecología y Manejo de Ecosistemas Acuáticos Pampeanos, Chascomús, Argentina: 42.

DRAGO, E. \& R. QUIRÓS. 1996. The hydrochemistry of the inland waters of Argentina: a review. International Journal of Salt Lake Research, 4: 315325.

EBERLEIN, K. \& G. KATTNER. 1987. Automatic method for the determination of orthophosphate and total dissolved phosphorous in the marine environment. Fresenius Zeichtung für Analytical Chemistry, 326: 354-357.

ECHANIZ, S. \& A. M. VIGNATTI. 1996. Cladoceros limnéticos de la provincia de La Pampa (Argentina). Revista de la Facultad de Agronomía. Universidad Nacional de La Pampa, La Pampa, Argentina. 1(9): 65-80.

ECHANIZ, S. \& A. M. VIGANTTI. 2002. Variación anual de la taxocenosis de cladóceros planctónicos (Crustacea: Brachiopoda) de una laguna de elevada salinidad (La Pampa, Argentina). Neotrópica, 48: 11-17.

ECHANIZ, S., A. M. VIGNATTI, J. JOSÉ DE PAGGI \& J. C. PAGGI. 2005. Riqueza y composición del zooplancton de la región pampeana argentina. Revista FABICIB, 9: 25-39.

ECHANIZ, S., A. M. VIGNATTI, S. JOSÉ DE PAGGI, J. C. PAGGI \& A. PILATI. 2006. Zooplankton seasonal abundance of South American saline shallow lakes. Int. Revue ges. Hydrobiol., 91: 86-100. 
FUSCHINI MEJÍA, M. C. 1994. El agua en las llanuras. UNESCO/ORCYT. Montevideo. Uruguay. $54 \mathrm{pp}$.

GERALDI, A. M. 2003. Hidrografía de la laguna Malaver. Tesis de Licenciatura, Universidad Nacional del Sur. Bahía Blanca. Argentina. 45 pp.

GRASSHOF, K. 1969. Un sistema de canal múltiple para análisis de nutrientes en aguas de mar con récord de datos analógicos y digitales. Avances en análisis automáticos. Technicon Internacional Symposium, Chicago. In: Manuel d'analyse des sels nutritifs dans l'eau de mer. Utilisation de l'autoanalyseur II Technicon. Treguer, P. \& P. Le Corre (eds.): 110 pp. $2^{\text {ème }}$ edition. Université de Bretagne Occidentale Brest. France.

GRASSHOF, K., M. EHRHARD \& K. KREMLING. 1983. Methods of seawater analysis. $2^{\text {nd }}$ ed. Weinheim. Germany: Verlag Chemie. 419 pp.

HALSE, S. A., R. J. SHIEL \& W. D. WILLIAMS. 1998. Aquatic invertebrate of Lake Gregory, northwestern Australia, in relation to salinity and ionic composition. Hydrobiologia, 381: 15-29.

IRIONDO, M. 2004. Large wetlands of South America: a model for Quaternary humid environments. Quaternary International, 114(1): 3-9.

JEPPESEN, E., M. SØNDERGAARD, E. MORTENSEN, P. KRISTENSEN, B. RIEMANN, H. J. JENSEN, J. P. MÜLLER, O. SORTKJÆR, J. P. JENSEN, K. CHRISTOFFERSEN, S. BOSSELMANN \& E. DALL, 1990. Fish manipulation as a lake restoration tool in shallow, eutrophic temperate lakes 1: cross-analysis of three Danish casestudies. Hydrobiologia, 200/201: 205-218.

JEPPESEN, E., P. KRISTENSEN, J. P. JENSEN, M. SØNDERGAARD, E. MORTENSENS \& T. LAURIDSEN. 1991. Recovery resilience following a reduction in external phosphorus loading of shallow, eutrophic Danish lakes: duration, regulating factors and methods for overcoming resilience. Memoria del Instituto Italiano di Idrobiologica, 48: 127-148.

JOSÉ DE PAGGI, S. B. \& J. C. PAGGI. 1998. Zooplancton de ambientes acuáticos con diferente estado trófico y salinidad. Neotrópica, 44(111112): 95-106.

JOSÉ DE PAGGI, S. B. \& J. C. PAGGI. 2007. Zooplankton. In: The middle Paraná River. Limnology of a Subtropical Wetland. Part 3: Chapter 9. Iriondo, M. H., J. C. Paggi \& M. J. Parma (eds.): 229-249. Springer Berlin, Heidelberg. Berlin, Germany.
JUNK, W. J. (Ed.). 1997. The Central Amazon Floodplain. Ecological Studies 126. Springer Verlag, Berlin, Germany: 525 pp.

LEWIS, W. M., S. HAMILTON \& J. SAUNDERS. III. 1995. Rivers of the northern South America. In: River and stream ecosystems. C. E. Cushing, K. W. Cummins \& G. W. Minshall (eds.): 219-256. Elsevier, Amsterdam, Holland.

LORENZEN, C. L. 1967. Determination of chlorophyll and pheopigments. Spectrophotometric equations. Limnol. Oceanogr., 12: 343-346.

MAIZELS, P., E. ETCHEPARE, E. CHORNOMAZ, J. BUSTINGORRY, R. ESCARAY \& V. CONZONNO. 2003. Parámetros abióticos y biomasa planctónica en la laguna Chascomús (Provincia de Buenos Aires). Período de inundación 2002. Biología Acuática, 20: 6-11.

MC NAUGHTON, S. J. 1968. Ecology structure and function in California grassland. Ecology, 49: 962972.

MELO, W. D. 1995. Comportamiento y dinámica del Arroyo Napostá Chico. Seminario de Geografía de los Recursos Naturales. Universidad Nacional del Sur. Bahía Blanca. Argentina. 74 pp.

MENESES JUNCO, L. 1997. Estructura de la comunidad de cladóceros en la laguna Alalay (Cochabamba, Bolivia). Revista Boliviana de Ecología, 3: 47-59.

MOSS, B. 1990. Engineering and biological approaches to the restoration from eutrophication of shallow lakes in which aquatic plant communities are important components. Hydrobiologia, 200/201: 367-378.

NIXON, S. W. 1981. Freshwater inputs and estuarine productivity. In: Proceedings of the National Symposium on Freshwater inflow to Estuaries. Cross, R. D. \& D. L. Williams (eds.): 31-57. Fish and Wildlife Service, Office of Biological Services. (FWS/OBS-81/04, Vol 1.), USA.

PAGGI, J. C. 1993. Análisis preliminar de la distribución geográfica de los cladóceros suramericanos. In: Conferencias de Limnología. A. Bôltovskoy \& H. L. López (eds.): 105-113. Instituto de Limnología "Dr. Raúl A. Ringuelet", La Plata, Argentina.

PAGGI, J. C. \& S. JOSÉ DE PAGGI. 1990. Zooplankton of the lotic and lentic environments of the Middle Paraná River. Acta Limnologica Brasiliensia, 3: 685-719.

PECORARI, S., S. JOSÉ DE PAGGI \& J. C. PAGGI. 2006. Assessment of the urbanization effect on a 
lake by zooplankton. Water Resources, 33(6): 677685.

PENNAK, R. W. 1978. Fresh-Water Invertebrates of the United States. $2^{\text {ond }}$ ed. Wiley Interscience Publication, New York, USA, 803 pp.

PILATI, A. 1997. Copépodos calanoideos de la Provincia de La Pampa. Revista de la Facultad de Agronomía, Universidad Nacional de La Pampa, 9(2): 57-67.

PILATI, A. 1999. Copépodos ciclopoideos en la Provincia de La Pampa (Argentina). Revista de la Facultad de Agronomía, Universidad Nacional de La Pampa, 10(1): 29-44.

QUIRÓS, R. 1991. Empirical relationships between nutrients, phytoplankton and zooplankton and relative fish biomass in lakes and reservoirs of Argentina. Verh. Internat. Verein. Limnol., 24: 11981206.

QUIRÓS, R. 1998. Classification and state of the environment of the Argentine Lakes. In: Study Report for the Lake Environment Conservation in Developing Countries. Chapter 2. Lakes of Argentina. International Lake Environment Committee Foundation (eds.): 30-50. Kusatsu, Japan.

QUIRÓS, R. 2002. The nitrogen to phosphorus ratio for lakes: A cause or a consequence of aquatic biology? In: El Agua en Iberoamerica: De la Limnología a la Gestión en Sudamerica. Fernández, A. C. \& G. M. Chalar (eds.): 11-26. Programa Iberoamericano de Ciencia y Tecnología para el Desarrollo (CYTED), Buenos Aires, Argentina.

QUIRÓS, R. \& E. DRAGO. 1999. The environmental state of Argentinean lakes: An overview. Lakes \& Reservoirs: Research and Management, 4: 5564.

QUIRÓS, R., J. J. ROSSO, A. M. RENNELLA, A. SOSNOVSKY \& M. BOVERI. 2002. Análisis del estado trófico de las lagunas pampeanas (Argentina). Interciencia, 27(11): 584-591.

RENNELLA, A. \& R. QUIRÓS. 2002. Relations between planktivorous fish and zooplankton in two very shallow lakes of the Pampa Plain. Verh. Internat. Verein. Limnol., 28: 887-891.

RENNELLA, A. \& R. QUIRÓS. 2006. The effects of hydrology on plankton biomass in shallow lakes of the Pampa Plain. Hydrobiologia, 556: 181-191.

RINGUELET, R. A. 1972. Ecología y biocenología del hábitat lagunar o lago de tercer orden de la Región Neotrópica templada (Pampasia sudoriental de la Argentina). Physis, 21(82): 55-75.

RINGUELET, R. A., I. MORENO \& E. FELDMAN. 1965. El Zooplancton de las lagunas de la Pampa deprimida y otras aguas superficiales de la Llanura Bonaerense (Argentina). Physis, 27(74): 187-200.

RINGUELET, R. A., A. SALIBIÁN, E. CLAVERIE, \& S. ILHERO. 1967. Limnología química de lagunas pampásicas (Provincia de Buenos Aires). Physis, 27(74): 201-221.

SCHEFFER, M. 1990. Multiplicity of stable states in freshwater systems. Hydrobiologia, 200/201: 475486.

SCHEFFER, M. 1998. Ecology of shallow lakes. London, UK: Champman \& Hall. 370 pp.

SCHEFFER, M., S. H. HOSPER, M. L. MEIJER, B. MOSS \& E. JEPPESEN. 1993. Alternative equilibria in shallow lakes. Trends Ecol. Evol., 8: 275279.

SHANNON, C. E. \& W. WEAVER. 1949. The mathematical theory of communications. Urbana, Illinois, USA. University Illinois Press. 117 pp.

SOSNOVSKY, A. \& R. QUIRÓS. 2006. El estado trófico de las pequeñas lagunas pampeanas (Argentina), su relación con la hidrología y la intensidad del uso de la tierra. Ecología Austral, 16: 115124.

TIMMS, B. V. 1998. Further studies on saline lakes of the eastern Paroo inland, New South Wales, Australia. Hydrobiologia, 381: 31-42.

VERVOOST, F. B. 1967. Las comunidades vegetales de la Depresión del Salado (Provincia de Buenos Aires). En: La vegetación de la República Argentina. Serie Fitogeográfica 7. Instituto Nacional de Tecnología Agropecuaria, Buenos Aires, Argentina, 219 pp.

VIGNATTI, A., S. ECHANIZ \& M. C. MARTÍN. 2007. El zooplancton de tres lagos someros de diferente salinidad y estado trófico en la región semiárida pampeana (Argentina). Gayana, 71(1): 34-48. 